\title{
Field evaluation of the influence of garlic extract and probiotic cultures on sows and growing pigs"
}

\author{
MARTA SATORA, ANNA RZASSA*, KRZYSZTOF RYPUŁA, \\ KATARZYNA PŁONECZKA-JANECZKO
}

\begin{abstract}
Department of Epizootiology and Clinic for Birds and Exotic Animals, Faculty of Veterinary Medicine, Wrocław University of Environmental and Life Sciences, Plac Grunwaldzki 45, Wrocław, Poland *Department of Immunology, Pathophysiology and Veterinary Preventive Medicine, Faculty of Veterinary Medicine, Wrocław University of Environmental and Life Sciences, Norwida 31, Wrocław, Poland
\end{abstract}

Satora M., Rząsa A., Rypuła K., Płoneczka-Janeczko K.

Field evaluation of the influence of garlic extract and probiotic cultures on sows and growing pigs Summary

The present study aimed to evaluate the influence of a garlic extract and probiotic strains, Enterococcus faecium, Lactobacillus rhamnosus and Lactobacillus fermentum, on sows and their offspring. A total of 24 sows and the 301 piglets they farrowed were used in this trial. The animals (sows and piglets) were assigned to 3 groups: group A, supplemented with garlic extract; group B, supplemented with probiotics; and the control group $\mathrm{C}$, without supplementation. The trial lasted from the $80^{\text {th }}$ day of gestation to the weaning day for sows. For piglets, results were analyzed during two periods, from the $8^{\text {th }}$ day to the $56^{\text {th }}$ or the $147^{\text {th }}$ day of life, in order to determine the more desirable duration of supplementation. Morphological and biochemical blood parameters, as well as body weight and piglet losses, were recorded. At weaning, the piglets supplemented with probiotics were heavier than the rest of experimental population $(P \leq \mathbf{0 . 0 1})$. On the day of sale for slaughter, the pigs from the control group were on average $15 \mathrm{~kg}$ lighter $(P \leq 0.01)$ than those from both supplemented groups. Differences in blood parameters were also noted between the groups. Our findings suggest that the use of a garlic extract and probiotic bacteria may help improve pig performance at various stages of the production cycle.

Keywords: pigs, garlic extract, probiotics, rearing results, blood parameters

The ban on antibiotic growth promoters (AGP) and the tendency towards a decrease in the therapeutic use of antibiotics prompted search alternative strategies $(24,25,34)$. Currently, various feed additives present very promising options. Because of their immunoprotective or immunostimulating properties, they can be given to pigs from different production groups, depending on farm conditions. Those with immunoprotective effects are involved in maintaining the organism's homeostasis and do not stimulate the host's immunological system. They include probiotics, immunoglobulins (derived from blood plasma, colostrum, or egg yolk) and bacteriophages, which can be administered from birth. Acidifiers could be introduced before weaning, and enzymes after weaning as well $(2,4,27-29)$. The role of immunostimulating additives is to activate the host's immune system, and that is why they are usually introduced around the weaning period or later. This

1) The research was financed/co-financed under the Leading Research Groups support project from a subsidy increased for the period 2020-2025 in the amount of $2 \%$ of the subsidy referred to in Art. 387 (3) of the Law of 20 July 2018 on Higher Education and Science, obtained in 2019. group of additives includes probiotics, symbiotics and, conditionally, bioplexes $(\mathrm{Zn}, \mathrm{Cu}, \mathrm{Mn}, \mathrm{Se})$, which are administered when an animal is lacking minerals. An interesting group of feed additives is also yeast, yeast derivatives and herbs that demonstrate immunoprotective and immunostimulating properties $(17,26,30)$.

Probiotics are most often identified on the basis of a live culture of microorganisms that improve the host's indigenous microbial balance $(9,36)$. Positive effects of the use of probiotics that increase the host's immunity to infectious agents include greater accessibility of nutrients, competitive inhibition of epithelial adherence, inhibition of epithelial invasion by pathogenic bacteria and production of antimicrobial substances $(1,9,21)$.

Natural bioactive compounds obtained from plants are referred to as phytobiotics, phytochemicals or phytogenics. Main attention is paid to their antimicrobial and antioxidant characteristics. Phytobiotics also have an immunomodulatory effect through the enhancement of immune cell proliferation, modulation of cytokines and antibody titers. Some authors suggest that they 
can be used as growth promoters instead of banned AGPs $(14,22)$. Garlic preparations have been used in medicine for many years. Most of them show a broad spectrum of antibacterial activity. Garlic inhibits the growth of Gram-negative and Gram-positive bacteria. It also exhibits antifungal activity, which is based on the inhibition of mycotoxin production (5).

Search for growth promoters that could optimise large-scale farming has continued incessantly since the ban on AGPs. However, an ideal substitute still has not been found, and research in this area seems to be much needed, especially under field conditions, since optimum nutrition, health and management are the basis of optimal productivity. Although many reports on the use of probiotics or garlic derivatives in pigs can be found in the literature, there is still little informa- tion on such dietary supplementation in practice. This field study was undertaken to evaluate the effects of garlic extract and cultures of Enterococcus faecium, Lactobacillus rhamnosus and Lactobacillus fermentum on sows and piglets that received them as feed additives in terms of their health status and production results, and to determine the optimal administration time and period for such preparations.

\section{Material and methods}

All procedures were approved by the Local Ethics Committee at the University of Environmental and Life Sciences, Wrocław, Poland (application No. 22/2014).

The study was performed on 24 crossbreed sows (Polish Large White $\times$ Polish Landrace) and their 301 offspring (Polish Large White $\times$ Polish Landrace $\times$ Pietrain) at a com-

Tab. 1. Composition and nutritive value of diets

\begin{tabular}{|c|c|c|c|c|c|c|}
\hline & $\begin{array}{l}\text { Empty and pregnant } \\
\text { sows }\end{array}$ & Lactating sows & Suckling piglets & Weaners & $\begin{array}{c}\text { Fatteners up to } \\
50 \mathrm{~kg}\end{array}$ & $\begin{array}{l}\text { Fatteners over } \\
\quad 50 \mathrm{~kg}\end{array}$ \\
\hline \multicolumn{7}{|l|}{ Ingredients [\%] } \\
\hline Barley & 50 & 50 & 27 & 25 & 18.5 & 19 \\
\hline Wheat & & 30 & 40 & 35 & 30 & 30 \\
\hline Triticale & 22.5 & & & & 30 & 30 \\
\hline Rye & 20 & & & & & \\
\hline Soybeans & 5 & 15 & 2.5 & 13.5 & 17 & 17 \\
\hline Fish meal & & & 2.8 & & & \\
\hline Soybean oil & & 0.7 & 2 & 1.5 & 1.8 & 1.3 \\
\hline Acidizer & & 0.3 & 0.3 & & 0.2 & 0.2 \\
\hline \multirow[t]{2}{*}{ Zinc oxide } & & & 0.4 & & & \\
\hline & Premixa 2.5 & Premix 4 & Concentrate $^{c} 25$ & Concentrate $^{\mathrm{d}} 25$ & Premix 2.5 & Premix 2.5 \\
\hline \multicolumn{7}{|l|}{ Nutritive value } \\
\hline Energy [MJ/kg] & 12.84 & 12.95 & 14.35 & 14.27 & 13.63 & 13.51 \\
\hline Total Protein $[\mathrm{g} / \mathrm{kg}]$ & 124.82 & 162.85 & 202.54 & 225.38 & 168.83 & 169.41 \\
\hline
\end{tabular}

Explanations: Composition of the mineral and vitamin mixture per $1 \mathrm{~kg}$ :

a Polfamix LP TOP1: Lysine 4 g, Methionine 2 g, Threonine 2 g, Total Calcium 297 g, Total Phosphate 69 g, Sodium 62 g, Magnesium 10 g, Vitamin A 480000 IE, Vitamin D3 65,000 IE, Vitamin E 3000 mg, Vitamin K 80 mg, Vitamin B1 80 mg, Vitamin B2 200 mg, Vitamin B6 $150 \mathrm{mg}$, Vitamin B12 $1200 \mathrm{mcg}$, Biotin 8,000 mcg, Niacin $1250 \mathrm{mg}$, Folic Acid $200 \mathrm{mg}$, Ca Pantothenate $600 \mathrm{mg}$, Choline 12,000 mg, Manganese 3100 mg, Zinc 5000 mg, Iron 4000 mg, Copper 990 mg, Iodine 30 mg, Selenium 12 mg, Betaine $5500 \mathrm{mg}$, Antioxidant $1000 \mathrm{mg}$;

${ }^{\mathrm{b}}$ Polfamix LK TOP2: Lysine $50 \mathrm{~g}$, methionine $7 \mathrm{~g}$, Threonine $8 \mathrm{~g}$, Total calcium $230 \mathrm{~g}$, Total Phosphorus $60 \mathrm{~g}$, Sodium $55 \mathrm{~g}$, Magnesium 10 g, Vitamin A 300000 IE, Vitamin D3 50000 IE, Vitamin E 3000 mg, Vitamin K 100 mg, Vitamin B1 55 mg, Vitamin B2 225 mg, Vitamin B6 $100 \mathrm{mg}$, Vitamin B12 $1000 \mathrm{mcg}$, Biotin 10,000 mcg, Niacin $700 \mathrm{mg}$, Folic Acid $50 \mathrm{mg}$, Ca Pantothenate $500 \mathrm{mg}$, Choline 12,000 mg, Manganese $2500 \mathrm{mg}$, Zinc $2500 \mathrm{mg}$, Iron $3000 \mathrm{mg}$, Copper $625 \mathrm{mg}$, Iodine $30 \mathrm{mg}$, Selenium $12 \mathrm{mg}$, Betaine $5500 \mathrm{mg}$; ${ }^{c}$ Vitarex PW3: Crude protein 400 g, Crude oils and fats $24.1 \mathrm{~g}$, Metabolic energy 12.43 MJ, Crude ash 140.2 g, Crude fibre 45.2 g, Lysine $37 \mathrm{~g}$, Methionine $16 \mathrm{~g}$, Methionine + cystine $22.05 \mathrm{~g}$, Threonine $18 \mathrm{~g}$, Tryptophan $5.35 \mathrm{~g}$, Calcium $30 \mathrm{~g}$, Phosphorus 13 g, Sodium 9.5 g, Magnesium 1.82 g, Vitamin A 48000 IU, Vitamin D3 8000 IU, Vitamin E 140 IU, Zinc mg 480 mg, Copper mg 400 mg; ${ }^{\mathrm{d}}$ Vitarex PW4: Crude protein $350 \mathrm{~g}$, Crude oils and fats $24 \mathrm{~g}$, Metabolic energy 11.65 MJ, Crude ash 170.2 g, Crude fibre 50.3 g, Lysine $33.2 \mathrm{~g}$, Methionine $9.7 \mathrm{~g}$, Methionine + cystine 14, $54 \mathrm{~g}$, Threonine $18 \mathrm{~g}$, Tryptophan $4.62 \mathrm{~g}$, Calcium $35 \mathrm{~g}$, Phosphorus $19.5 \mathrm{~g}$, Sodium 8.5 g, Magnesium 2.02 g, Vitamin A 60000 IU, Vitamin D3 8000 IU, Vitamin E 200 IU, Zinc mg 500 mg, copper mg 604 mg; e Polfamix PT1: Lysine 90 g, Methionine 17 g, Threonine 19 g, Total Calcium 254 g, Total Phosphorus 70 g, Sodium 67 g, Magnesium 8 g, Vitamin A 260000 IE, Vitamin D3 70,000 IE, Vitamin E 3,000 mg, Vitamin K 70 mg, Vitamin B1 55 mg, Vitamin B2 200 mg, Vitamin B6 $80 \mathrm{mg}$, Vitamin B12 $1200 \mathrm{mcg}$, Biotin $6000 \mathrm{mcg}$, Niacin $800 \mathrm{mg}$, Folic Acid $20 \mathrm{mg}$, Ca Pantothenate $500 \mathrm{mg}$, Choline $6000 \mathrm{mg}$, Manganese $1000 \mathrm{mg}$, Zinc 4000 mg, Iron $3000 \mathrm{mg}$, Copper 980 mg, Iodine 25 mg, Selenium 12 mg;

${ }^{f}$ Polfamix PT2: Lysine 80 g, Methionine $10 \mathrm{~g}$, Threonine $14 \mathrm{~g}$, Total Calcium $245 \mathrm{~g}$, Total Phosphate $69 \mathrm{~g}$, Sodium $66 \mathrm{~g}$, Magnesium 8 g, Vitamin A 260000 IE, Vitamin D3 60,000 IE, Vitamin E 3,000 mg, Vitamin K 60 mg, Vitamin B1 50 mg, Vitamin B2 150 mg, Vitamin B6 65 mg, Vitamin B12 $1200 \mathrm{mcg}$, Biotin $2000 \mathrm{mcg}$, Niacin $750 \mathrm{mg}$, Folic Acid $15 \mathrm{mg}$, Ca of Pantothenate $500 \mathrm{mg}$, Choline $5000 \mathrm{mg}$, Manganese $1000 \mathrm{mg}$, Zinc $3000 \mathrm{mg}$, Iron $2000 \mathrm{mg}$, Copper $900 \mathrm{mg}$, Iodine $25 \mathrm{mg}$, Selenium $10 \mathrm{mg}$, Iron $3000 \mathrm{mg}$, Copper $980 \mathrm{mg}$, Iodine $25 \mathrm{mg}$, Selenium $12 \mathrm{mg}$, Antioxidant $1000 \mathrm{mg}$. 
mercial farm. The farm operates in a closed cycle with a oneweek production rhythm, and the basic herd consists of 130 sows. Animals included in the study received routine care and veterinary treatment at the farm. After the completion of the study, sows were kept for breeding, and fatteners were intended for sale. Two weeks before mating, sows were vaccinated with Eryseng Parvo (Laboratories Hipra S.A., Spain) and Porcilis PRRS (Intervet, Netherlands), and 4 months later the Porcilis PRRS vaccination was repeated. Additionally, 2 weeks before parturition, Biotropina $^{\circledR}$ (Biowet Drwalew S.A., Poland) was administered to sows. Iron in the form of Ferran 200 (Vet-Agro sp. z o.o., Poland) was given to piglets on the $2^{\text {nd }}$ day of life in a dose of $1 \mathrm{ml} /$ animal as an intramuscular injection. The piglets were vaccinated against Porcine Circovirus-2 with Circovac (Merial, France) on the $21^{\text {st }}$ day of life.

After weaning, sows were moved to the reproduction sector and kept in individual unbedded pens until gestation was confirmed, and then they were moved to group bedded pens with a feed station for up to 30 heads (Wolbrink System NEDAP, Netherlands). On the $80^{\text {th }}$ day of gestation, 24 multiparous sows from one technological group were randomly chosen for the trial and divided into 3 groups: $\mathrm{C}$ - control, $\mathrm{A}$ - fed with garlic extract, and B - fed with a composition of probiotic bacteria. All sows were in a similar body condition, and they were between the $2^{\text {nd }}$ and $5^{\text {th }}$ parities. Only a negative control was included in the study. A positive control (sows or piglets supplemented with antibiotics) was not included in this research because of the EU-wide ban on the use of antibiotics as antimicrobial growth promoters (AGP) in animal feed (introduced on January 1, 2006).

Sows from Groups A and B were individually administered additives from the $80^{\text {th }}$ day of gestation to the weaning day. Piglets were housed with their dams in triple-slatted pens in the farrowing unit. All piglets were divided into groups like the sows.

After weaning (on the $23^{\text {rd }}$ day of life) piglets were moved to the nursery unit and kept in grouped slatted pens for 20 weaners. At the $11^{\text {th }}$ week of life, they were moved to the fattening sector and kept in grouped deep-litter pens for 50 fatteners.

Animals were fed with mixtures prepared at the farm. Feed mixture formulas for all production groups are presented in Table 1 . The scheme of the study is shown in Tables 2 and 3. In Table 3, the labels $\mathrm{B}+$ and $\mathrm{A}+$ denote the supplementation of additives to piglets from the $8^{\text {th }}$ day of life to the end of the trial (for 147 days) whereas B- and A- mean that the additives were administered up to the $56^{\text {th }}$ day of life. Garlic extract and probiotic bacteria were given to each animal individually by direct oral administration. Allivet ${ }^{\mathrm{TM}}$ (Centaur, Poland, authorization number PL2408001p) is a complementary feed composed of $6: 1$ garlic extract, which means that one litre of Allivet is obtained from $6 \mathrm{~kg}$ of garlic. The preparation contains more than 100 allyl sulfides, and one of them is allicin in a concentration below $1 \%$.

Strains of the probiotic bacteria Enterococcus faecium CCM 6226 (serial number 030714), Lactobacillus rhamnosus CCM 1825 (serial number 010914) and Lactobacillus
Tab. 2. Study scheme for sows

\begin{tabular}{|c|c|}
\hline $\begin{array}{l}\text { Group C } \\
(n=8)\end{array}$ & Control sows with standard feeding \\
\hline $\begin{array}{l}\text { Group B } \\
(n=8)\end{array}$ & $\begin{array}{l}\text { Standard feeding with the addition of probiotic bacteria } \\
\text { Enterococcus faecium, Lactobacillus rhamnosus and } \\
\text { Lactobacillus fermentum; } \\
2 \mathrm{~g} / \mathrm{sow} / \mathrm{day} \text { from the } 80^{\text {th }} \text { day of gestation to the weaning day }\end{array}$ \\
\hline $\begin{array}{l}\text { Group A } \\
(n=8)\end{array}$ & $\begin{array}{l}\text { Standard feeding with the addition of garlic extract; } \\
10 \mathrm{ml} / \mathrm{sow} \text { every } 3 \text { days from the } 80^{\text {th }} \text { day of gestation to the } \\
\text { weaning day }\end{array}$ \\
\hline
\end{tabular}

Tab. 3. Study scheme for offspring. Within experimental groups $\mathrm{B}+$ and $\mathrm{A}+$ on the $56^{\text {th }}$ day; litters were divided into 2 subgroups: $\mathrm{B}+$ and $\mathrm{B}-, \mathrm{A}+$ and $\mathrm{A}-$

\begin{tabular}{|c|c|}
\hline $\begin{array}{l}\text { Group C } \\
(\mathrm{n}=90)\end{array}$ & Control pigs with standard feeding \\
\hline $\begin{array}{l}\text { Group B+ } \\
(n=42)\end{array}$ & $\begin{array}{l}\text { Standard feeding with the addition of probiotic bacteria from } \\
\text { the } 8^{\text {th }} \text { day of life to the end of the experiment; } 2 \mathrm{~g} / \text { day/head }\end{array}$ \\
\hline $\begin{array}{l}\text { Group B- } \\
(n=42)\end{array}$ & $\begin{array}{l}\text { Standard feeding with the addition of probiotic bacteria from } \\
\text { the } 8^{\text {th }} \text { day of life to the } 56^{\text {th }} \text { day of life; } 2 \mathrm{~g} / \text { day/head }\end{array}$ \\
\hline $\begin{array}{l}\text { Group A+ } \\
(n=46)\end{array}$ & $\begin{array}{l}\text { Standard feeding with the addition of garlic extract from the } \\
8^{\text {th }} \text { day of life to the end of the experiment; } 1 \text { ml every } 3 \text { days } \\
\text { per } 10 \mathrm{~kg} \text { of body weight }\end{array}$ \\
\hline $\begin{array}{l}\text { Group A- } \\
(n=46)\end{array}$ & $\begin{array}{l}\text { Standard feeding with the addition of garlic extract from the } \\
8^{\text {th }} \text { day of life to the } 56^{\text {th }} \text { day; } 1 \mathrm{ml} \text { every } 3 \text { days per } 10 \mathrm{~kg} \text { of } \\
\text { body weight }\end{array}$ \\
\hline
\end{tabular}

fermentum CCM 7192 (serial number 011014) were selected from a collection of microorganisms of PharmaGal-Bio (Slovakia). All strains were admitted to trading as feed additives (EC Regulation 1831/2003) and are on the updated list of 24.10.2018. Every kilogram of the supplemented feed comprised $690 \mathrm{~g}$ of corn starch, $50 \mathrm{~g}$ of maltodextrin, $250 \mathrm{~g}$ of protein and $10 \mathrm{~g}$ of the probiotic bacteria $\left(1 \times 10^{9}\right.$ $\mathrm{CFU} / \mathrm{g}$ ). Before administration, $2 \mathrm{~g}$ of this mixture was suspended in $10 \mathrm{ml}$ of water. The strains were selected on the basis of positive results reported in numerous publications for each strain $(6,7,40)$.

Data and sample collection. Sows: Blood samples were collected via venepuncture of the vena cava cranialis into 2 tubes containing K EDTA (dipotassium salt of ethylenediaminetetra-acetic acid) as an anticoagulant and with polypropylene pearls to obtain serum (on the $3^{\text {rd }}$ day before parturition).

Offspring: Body weight was estimated on the $2^{\text {nd }}$ (start of the experiment), $23^{\text {rd }}$ (weaning), $56^{\text {th }}$ (additional regrouping of piglets depending on feed additive supplementation) and $147^{\text {th }}$ days of life (sale). Because of the feeding technology used at the farm (feed for each production group was stored in an individual silo) and the fact that piglets were fed $\mathrm{ad}$ libidum, FCR (feed conversion ratio) was not calculated in this trial. Losses and health disorders were recorded. Blood samples were collected via venepuncture of the vena cava cranialis into 2 tubes containing $\mathrm{K}_{2}$ EDTA and polypropylene pearls during the $1^{\text {st }}, 3^{\text {rd }}, 4^{\text {th }}, 8^{\text {th }}$ and $11^{\text {th }}$ weeks of life.

Laboratory analysis. A morphological analysis was performed using a CELL-DYN 3700 analyser, manufactured by Abbot Laboratories, Polska Sp. z o. o. The following parameters were determined: total number of white blood cells (WBC), neutrophils (NEU), lymphocytes 
(LYM), monocytes (MONO), eosinophils (EOS), basophils (BASO), red blood cell count (RBC), haemoglobin (HGB), haematocrit (HCT), mean red cell volume (MCV), mean corpuscular haemoglobin $(\mathrm{MCH})$, mean corpuscular haemoglobin concentration (MCHC) and platelet count (PLT). Total serum protein (TP) and its fractions: albumins (ALB), alpha-globulins $(\alpha)$, beta-globulins $(\beta)$, gamma-globulins $(\gamma)$, as well as the albumin to globulin ratio, were estimated on SAS-MX Serum Protein SB agar gels (Helena Bio-

Tab. 4. The average body weight of piglets during the experiment $(\bar{x} \pm$ SD)

\begin{tabular}{|c|c|c|c|c|c|}
\hline \multirow{2}{*}{ Day of life } & \multicolumn{4}{|c|}{ Group } \\
& C & \multicolumn{2}{|c|}{ B+ } & \multicolumn{2}{c|}{ A+ } \\
\hline \multirow{2}{*}{2} & 1.70 & \multicolumn{2}{|c|}{1.85} & \multicolumn{2}{c|}{1.73} \\
& \pm 0.22 & \multicolumn{2}{|c|}{ \pm 0.23} & \multicolumn{2}{|c|}{ \pm 0.21} \\
\multirow{3}{*}{23} & $7.41^{\mathrm{B}}$ & \multicolumn{2}{|c|}{$7.92^{\mathrm{A}}$} & \multicolumn{2}{|c|}{$7.25^{\mathrm{B}}$} \\
& \pm 0.72 & \multicolumn{2}{|c|}{ \pm 0.81} & \multicolumn{2}{c|}{ \pm 0.72} \\
\cline { 2 - 7 } & $\mathrm{C}$ & $\mathrm{B}+$ & $\mathrm{B}-$ & $\mathrm{A}+$ & $\mathrm{A}-$ \\
\hline \multirow{3}{*}{56} & $24.95^{\mathrm{B}}$ & $29.99^{\mathrm{AC}}$ & $28.08^{\mathrm{AD}}$ & $28.97^{\mathrm{A}}$ & $28.03^{\mathrm{AD}}$ \\
& \pm 1.43 & \pm 1.67 & \pm 1.71 & \pm 1.31 & \pm 1.53 \\
& $104.08^{\mathrm{B}}$ & $119.02^{\mathrm{A}}$ & $121.19^{\mathrm{A}}$ & $119.04^{\mathrm{A}}$ & $119.08^{\mathrm{A}}$ \\
& \pm 12.58 & \pm 9.01 & \pm 7.81 & \pm 9.31 & \pm 9.32 \\
\hline
\end{tabular}

Explanation: Significant differences are marked with pairs of letters ab, cd; small letters at $\mathrm{P} \leq 0.05$, and capital letters at $\mathrm{P} \leq 0.01$ sciences, Europe) with an ELITE 3000 Power Supply (by WEALTE) and in an SAS-MX chamber.

Statistical analysis. Supplementation results were compared by analysing variance procedures for a completely randomised design using the Statistica 12.5 statistical package (StatSoft Inc., Tulsa, OK). Differences between means were determined by the post-hoc Tukey test at 0.05 and 0.01 levels of significance. The results are presented in tables as the mean $(\overline{\mathrm{x}})$ and standard deviation $( \pm \mathrm{SD})$.

Tab. 5. White blood cells (WBC) in sows 3 weeks before delivery $(\bar{x} \pm$ SD)

\begin{tabular}{|l|c|c|c|}
\hline \multicolumn{1}{|c|}{ Parameter } & Group C & Group B+ & Group A+ \\
\hline \multirow{2}{*}{ WBC [g/l] } & $12.09^{\mathrm{a}}$ & $12.55^{\mathrm{a}}$ & $8.98^{\mathrm{b}}$ \\
& \pm 1.23 & \pm 2.50 & \pm 2.83 \\
NEU [g/l] & $5.84^{\mathrm{A}}$ & $5.28^{\mathrm{A}}$ & $3.36^{\mathrm{B}}$ \\
& \pm 0.71 & \pm 1.25 & \pm 1.05 \\
LYM [g/l] & 4.22 & 5.49 & 4.14 \\
& \pm 0.50 & $\pm 1,15$ & \pm 1.54 \\
MONO [g/l] & $0.92^{\mathrm{B}}$ & $1.07^{\mathrm{A}}$ & $0.84^{\mathrm{B}}$ \\
& \pm 0.001 & \pm 0.14 & \pm 0.07 \\
EOS [g/l] & 0.71 & 0.61 & 0.57 \\
& \pm 0.28 & \pm 0.18 & \pm 0.41 \\
BASO [g/l] & $0.03^{\mathrm{b}}$ & $0.07^{\mathrm{a}}$ & $0.04^{\mathrm{b}}$ \\
& \pm 0.00 & \pm 0.04 & \pm 0.00 \\
\hline
\end{tabular}

Explanation: as in Tab. 4.

Tab. 6. Morphological blood indicators for piglets in their $1^{\text {st }}, 3^{\text {rd }}$ and $4^{\text {th }}$ weeks of life $(\bar{x} \pm$ SD)

\begin{tabular}{|c|c|c|c|c|c|c|c|c|c|}
\hline \multirow{3}{*}{ Parameter } & \multicolumn{3}{|c|}{ Group C } & \multirow{2}{*}{\multicolumn{3}{|c|}{$\begin{array}{c}\text { Group B+ } \\
\text { Week of life }\end{array}$}} & \multirow{2}{*}{\multicolumn{3}{|c|}{ Group A+ }} \\
\hline & & & & & & & & & \\
\hline & $1^{\text {st }}$ & $3^{\text {rd }}$ & $4^{\text {th }}$ & $1^{\text {st }}$ & $3^{\text {rd }}$ & $4^{\text {th }}$ & $1^{\mathrm{st}}$ & $3^{\text {rd }}$ & $4^{\text {th }}$ \\
\hline WBC $[\mathrm{g} / \mathrm{l}]$ & $\begin{array}{l}11.56^{\mathrm{aA}} \\
\pm 2.30\end{array}$ & $\begin{array}{l}12.52^{\text {aA }} \\
\pm 3.02\end{array}$ & $\begin{array}{l}13.53^{\mathrm{a}} \\
\pm 5.33\end{array}$ & $\begin{array}{l}8.79^{\text {bd }} \\
\pm 2.39\end{array}$ & $\begin{array}{c}8.34^{b} \\
\pm 1.94\end{array}$ & $\begin{array}{c}7.72^{\mathrm{b}} \\
\pm 2.76\end{array}$ & $\begin{array}{l}5.43^{\mathrm{CB}} \\
\pm 1.45\end{array}$ & $\begin{array}{r}7.28^{8} \\
\pm 2.90\end{array}$ & $\begin{array}{c}9.29 \\
\pm 2.58\end{array}$ \\
\hline NEU [g/l] & $\begin{array}{l}8.56^{\mathrm{a}} \\
\pm 2.11\end{array}$ & $\begin{array}{r}4.25^{\mathrm{a}} \\
\pm 2.65\end{array}$ & $\begin{array}{r}4.28^{\mathrm{A}} \\
\pm 1.07\end{array}$ & $\begin{array}{l}5.62^{\text {bd }} \\
\pm 1.67\end{array}$ & $\begin{array}{l}1.48^{\mathrm{b}} \\
\pm 0.64\end{array}$ & $\begin{array}{l}1.95^{\mathrm{B}} \\
\pm 0.94\end{array}$ & $\begin{array}{l}2.82^{\mathrm{bc}} \\
\pm 1.07\end{array}$ & $\begin{array}{c}2.89 \\
\pm 1.14\end{array}$ & $\begin{array}{l}2.40^{\mathrm{B}} \\
\pm 0.59\end{array}$ \\
\hline LYM [g/l] & $\begin{array}{c}2.00 \\
\pm 0.41\end{array}$ & $\begin{array}{r}6.96^{\mathrm{A}} \\
\pm 1.85\end{array}$ & $\begin{array}{c}6.10 \\
\pm 1.97\end{array}$ & $\begin{array}{c}2.00 \\
\pm 0.59\end{array}$ & $\begin{array}{l}6.28^{\mathrm{A}} \\
\pm 0.99\end{array}$ & $\begin{array}{c}4.66 \\
\pm 2.93\end{array}$ & $\begin{array}{c}1.53 \\
\pm 0.29\end{array}$ & $\begin{array}{l}3.24^{\mathrm{B}} \\
\pm 1.50\end{array}$ & $\begin{array}{c}5.79 \\
\pm 2.78\end{array}$ \\
\hline MONO [g/l] & $\begin{array}{c}0.86 \\
\pm 0.31\end{array}$ & $\begin{array}{l}1.22^{\mathrm{a}} \\
\pm 0.68\end{array}$ & $\begin{array}{l}1.14^{\mathrm{A}} \\
\pm 0.38\end{array}$ & $\begin{array}{c}0.99 \\
\pm 0.27\end{array}$ & $\begin{array}{c}0.56^{b} \\
\pm 0.23\end{array}$ & $\begin{array}{l}0.48^{\mathrm{BB}} \\
\pm 0.25\end{array}$ & $\begin{array}{c}0.93 \\
\pm 0.48\end{array}$ & $\begin{array}{c}0.73 \\
\pm 0.17\end{array}$ & $\begin{array}{c}0.95^{\mathrm{a}} \\
\pm 0.28\end{array}$ \\
\hline EOS [g/l] & $\begin{array}{c}0.05 \\
\pm 0.02\end{array}$ & $\begin{array}{c}0.08 \\
\pm 0.06\end{array}$ & $\begin{array}{l}0.15^{\mathrm{a}} \\
\pm 0.07\end{array}$ & $\begin{array}{c}0.12 \\
\pm 0.08\end{array}$ & $\begin{array}{c}0.08 \\
\pm 0.06\end{array}$ & $\begin{array}{l}0.06^{\mathrm{b}} \\
\pm 0.03\end{array}$ & $\begin{array}{c}0.08 \\
\pm 0.06\end{array}$ & $\begin{array}{c}0.08 \\
\pm 0.04\end{array}$ & $\begin{array}{c}0.10 \\
\pm 0.05\end{array}$ \\
\hline BASO [g/l] & $\begin{array}{c}0.10 \\
\pm 0.03\end{array}$ & $\begin{array}{l}0.10^{A} \\
\pm 0.04\end{array}$ & $\begin{array}{c}0.05 \\
\pm 0.02\end{array}$ & $\begin{array}{c}0.07 \\
\pm 0.02\end{array}$ & $\begin{array}{l}0.02^{\mathrm{B}} \\
\pm 0.01\end{array}$ & $\begin{array}{c}0.04 \\
\pm 0.02\end{array}$ & $\begin{array}{c}0.08 \\
\pm 0.02\end{array}$ & $\begin{array}{l}0.05^{\mathrm{B}} \\
\pm 0.02\end{array}$ & $\begin{array}{c}0.07 \\
\pm 0.05\end{array}$ \\
\hline RBC [T/l] & $\begin{array}{c}4.29 \\
\pm 0.32\end{array}$ & $\begin{array}{l}5.29^{\mathrm{A}} \\
\pm 0.46\end{array}$ & $\begin{array}{c}5.67 \\
\pm 0.69\end{array}$ & $\begin{array}{c}3.68 \\
\pm 0.65\end{array}$ & $\begin{array}{c}4.02^{\mathrm{B}} \\
\pm 0.53\end{array}$ & $\begin{array}{c}5.30 \\
\pm 0.77\end{array}$ & $\begin{array}{c}3.92 \\
\pm 0.79\end{array}$ & $\begin{array}{c}4.49 \\
\pm 0.86\end{array}$ & $\begin{array}{c}5.39 \\
\pm 0.68\end{array}$ \\
\hline HGB [g/l] & $\begin{array}{c}8.81 \\
\pm 4.82\end{array}$ & $\begin{array}{l}105.77 \\
\pm 5.76\end{array}$ & $\begin{array}{l}110.75 \\
\pm 4.17\end{array}$ & $\begin{array}{c}70.78 \\
\pm 11.99\end{array}$ & $\begin{array}{l}101.85 \\
\pm 7.15\end{array}$ & $\begin{array}{l}114.21 \\
\pm 13.46\end{array}$ & $\begin{array}{c}76.64 \\
\pm 13.70\end{array}$ & $\begin{array}{c}97.85 \\
\pm 10.70\end{array}$ & $\begin{array}{l}110.71 \\
\pm 7.62\end{array}$ \\
\hline HCT [I//] & $\begin{array}{l}0.29^{a} \\
\pm 0.02\end{array}$ & $\begin{array}{c}0.36 \\
\pm 0.02\end{array}$ & $\begin{array}{c}0.38 \\
\pm 0.03\end{array}$ & $\begin{array}{l}0.24^{\mathrm{b}} \\
\pm 0.04\end{array}$ & $\begin{array}{c}0.33 \\
\pm 0.03\end{array}$ & $\begin{array}{c}0.38 \\
\pm 0.04\end{array}$ & $\begin{array}{c}0.26 \\
\pm 0.05\end{array}$ & $\begin{array}{c}0.32 \\
\pm 0.05\end{array}$ & $\begin{array}{c}0.38 \\
\pm 0.03\end{array}$ \\
\hline MCV [pg] & $\begin{array}{l}66.83 \\
\pm 3.93\end{array}$ & $\begin{array}{l}69.17^{\mathrm{B}} \\
\pm 3.24\end{array}$ & $\begin{array}{l}67.26^{b} \\
\pm 3.13\end{array}$ & $\begin{array}{l}64.76 \\
\pm 2.91\end{array}$ & $\begin{array}{l}82.29^{A} \\
\pm 8.77\end{array}$ & $\begin{array}{l}72.19^{a} \\
\pm 3.57\end{array}$ & $\begin{array}{l}66.63 \\
\pm 2.87\end{array}$ & $\begin{array}{l}72.02^{\mathrm{B}} \\
\pm 4.38\end{array}$ & $\begin{array}{l}70.21 \\
\pm 3.62\end{array}$ \\
\hline MCH [g/l] & $\begin{array}{r}19.33 \\
\pm 1.09\end{array}$ & $\begin{array}{l}20.04^{\mathrm{B}} \\
\pm 0.94\end{array}$ & $\begin{array}{l}19.57^{\mathrm{B}} \\
\pm 1.41\end{array}$ & $\begin{array}{l}19.16 \\
\pm 1.01\end{array}$ & $\begin{array}{l}25.99^{A} \\
\pm 3.01\end{array}$ & $\begin{array}{l}21.66^{\mathrm{A}} \\
\pm 0.89\end{array}$ & $\begin{array}{l}19.61 \\
\pm 0.75\end{array}$ & $\begin{array}{l}22.01^{\mathrm{B}} \\
\pm 1.79^{-}\end{array}$ & $\begin{array}{l}20.70 \\
\pm 1.28\end{array}$ \\
\hline MCHC [g/l] & $\begin{array}{l}289.5 \\
\pm 5.07\end{array}$ & $\begin{array}{c}290^{\circ \mathrm{B}} \\
\pm 5.61\end{array}$ & $\begin{array}{l}291.37 \\
\pm 15.22\end{array}$ & $\begin{array}{l}295.75 \\
\pm 7.83\end{array}$ & $\begin{array}{l}312.75^{\mathrm{A}} \\
\pm 10.47\end{array}$ & $\begin{array}{l}300.38 \\
\pm 4.87\end{array}$ & $\begin{array}{l}294.25 \\
\pm 2.82\end{array}$ & $\begin{array}{l}305.75^{\mathrm{a}} \\
\pm 17.50\end{array}$ & $\begin{array}{l}294.63 \\
\pm 7.07\end{array}$ \\
\hline PLT [g/l] & $\begin{array}{c}320.63 \\
\pm 132.02\end{array}$ & $\begin{array}{c}400.75^{\mathrm{a}} \\
\pm 161.53\end{array}$ & $\begin{array}{c}242.95 \\
\pm 102.25\end{array}$ & $\begin{array}{c}282.5 \\
\pm 117.78\end{array}$ & $\begin{array}{l}170.62^{\mathrm{bB}} \\
\pm 66.17\end{array}$ & $\begin{array}{c}253.96 \\
\pm 131.95\end{array}$ & $\begin{array}{r}326.88 \\
\pm 78.93\end{array}$ & $\begin{array}{c}424^{\mathrm{A}} \\
\pm 170.91\end{array}$ & $\begin{array}{c}374.25 \\
\pm 210.06\end{array}$ \\
\hline
\end{tabular}

Explanation: as in Tab. 4. 


\section{Results and discussion}

The average body weight on the $2^{\text {nd }}$ day of life was nearly equal in all groups and amounted to $1.76 \mathrm{~kg}$ (Tab. 4). On the weaning day, considerable differences in the body weight were noted between groups. Piglets from Group B+ were heavier than the rest of experimental population $(\mathrm{P} \leq 0.01)$. On days 56 and 147 , pigs from Group $\mathrm{C}$ were the lightest, which was confirmed statistically. During the fattening period, daily gains were noticeably higher in Group B-, which did not receive supplementation from day 56 . Both groups $\mathrm{A}(\mathrm{A}+, \mathrm{A}-)$ showed the same growth as $\mathrm{B}+$. The average number of piglets in experimental litters was similar, and amounted to 12.5. Piglet losses in groups $\mathrm{A}+, \mathrm{B}+$ and $\mathrm{C}$ were respectively $9.6 \%, 10.1 \%, 5.0 \%$ by the weaning day and another $2.1 \%, 5.6 \%, 3.2 \%$ by the day of transfer to the fattening sector. There were no losses during the fattening period. Throughout the trial, the pigs were in good health and did not require additional treatment.

The morphological blood analysis in sows confirmed statistically significant results only in the white blood cell patterns (Tab. 5).

Tab. 6 presents the morphological blood indicators for piglets in the $1^{\text {st }}, 3^{\text {rd }}$ and $4^{\text {th }}$ weeks of life. There were considerable differences in the WBC patterns for piglets: the highest number of WBC was always found in Group $C$, which differed from the other groups $(\mathrm{P} \leq 0.05, \mathrm{P} \leq 0.01)$. The same tendency was observed for NEU, LYM and MONO, but with smaller differentiation. Few differences were found between the groups for EOS and BASO. Similarly, for RBC, HGB and HCT on control days, the highest values were found in Group C, but statistically confirmed differences were noted only for RBC and HTC. The opposite relationship was observed for MCV, MCH and MCHC: the average value of these parameters was the lowest for control piglets. The lowest value of PLT was found in the $3^{\text {rd }}$ week in Group $\mathrm{B}+$, and it differed significantly from those in Groups $\mathrm{A}+(\mathrm{P} \leq 0.01)$ and $\mathrm{C}(\mathrm{P} \leq 0.05)$.

Tab. 7 presents morphological blood indicators of weaners in the $8^{\text {th }}$ and $11^{\text {th }}$ weeks of life. The concentration of WBC in the first control term was highest in Group $\mathrm{B}+$, and this result differed significantly from the WBC count in Group $\mathrm{C}(\mathrm{P} \leq 0.05)$. The count of WBC in the second control term was highest in Group $\mathrm{B}-$, which differed significantly from Groups $\mathrm{C}, \mathrm{A}+$, $\mathrm{A}-$ and $\mathrm{B}+(\mathrm{P} \leq 0.01$ and $\mathrm{P} \leq 0.05)$. A detailed analysis of NEU, LYM and MONO as well as other morphological blood parameters is presented in Table 7. The

Tab. 7. Morphological blood indicators for weaners in their $8^{\text {th }}$ and $11^{\text {th }}$ weeks of life $(\bar{x} \pm$ SD)

\begin{tabular}{|c|c|c|c|c|c|c|c|c|c|c|}
\hline \multirow[t]{2}{*}{ Parameter } & \multicolumn{2}{|c|}{ Group C } & \multicolumn{2}{|c|}{ Group B+ } & \multicolumn{2}{|c|}{$\begin{array}{c}\text { Group B- } \\
\text { Week of life }\end{array}$} & \multicolumn{2}{|c|}{ Group A+ } & \multicolumn{2}{|c|}{ Group A- } \\
\hline & $8^{\text {th }}$ & $11^{\text {th }}$ & $8^{\text {th }}$ & $11^{\text {th }}$ & $8^{\text {th }}$ & $11^{\text {th }}$ & $8^{\text {th }}$ & $11^{\text {th }}$ & $8^{\text {th }}$ & $11^{\text {th }}$ \\
\hline WBC [g/l] & $\begin{array}{l}15.95^{\mathrm{b}} \\
\pm 4.21\end{array}$ & $\begin{array}{l}17.68^{8} \\
\pm 1.60\end{array}$ & $\begin{array}{l}23.60^{\mathrm{a}} \\
\pm 7.94\end{array}$ & $\begin{array}{l}17.98^{b} \\
\pm 1.92\end{array}$ & $\begin{array}{l}21.93 \\
\pm 2.13\end{array}$ & $\begin{array}{c}20.70^{\text {aA }} \\
\pm 1.65\end{array}$ & $\begin{array}{l}18.08 \\
\pm 0.96\end{array}$ & $\begin{array}{l}17.53^{\mathrm{B}} \\
\pm 1.75\end{array}$ & $\begin{array}{l}19.63 \\
\pm 3.03\end{array}$ & $\begin{array}{l}17.60^{\mathrm{B}} \\
\pm 1.46\end{array}$ \\
\hline NEU [g/l] & $\begin{array}{l}7.15^{\mathrm{BB}} \\
\pm 1.46\end{array}$ & $\begin{array}{c}7.48 \\
\pm 1.13\end{array}$ & $\begin{array}{l}10.49^{\mathrm{ac}} \\
\pm 2.47\end{array}$ & $\begin{array}{c}7.24 \\
\pm 1.19\end{array}$ & $\begin{array}{l}12.18^{\mathrm{cA}} \\
\pm 2.03\end{array}$ & $\begin{array}{c}8.01 \\
\pm 1.35\end{array}$ & $\begin{array}{l}7.43^{\mathrm{BB}} \\
\pm 2.65\end{array}$ & $\begin{array}{c}6.50 \\
\pm 0.32\end{array}$ & $\begin{array}{l}8.89^{d} \\
\pm 0.32\end{array}$ & $\begin{array}{c}7.11 \\
\pm 1.73\end{array}$ \\
\hline LYM [g/l] & $\begin{array}{c}5.82^{\mathrm{b}} \\
\pm 1.88\end{array}$ & $\begin{array}{c}7.50^{\mathrm{B}} \\
\pm 1.38\end{array}$ & $\begin{array}{c}9.56^{\mathrm{a}} \\
\pm 3.55\end{array}$ & $\begin{array}{c}8,47 \\
\pm 1.35\end{array}$ & $\begin{array}{c}6.85 \\
\pm 0.35\end{array}$ & $\begin{array}{c}10.92^{\mathrm{a}} \\
\pm 1.08\end{array}$ & $\begin{array}{c}8.84 \\
\pm 2.35\end{array}$ & $\begin{array}{c}8.68 \\
\pm 1.71\end{array}$ & $\begin{array}{c}8.17 \\
\pm 2.75\end{array}$ & $\begin{array}{l}8.13^{b} \\
\pm 2.52\end{array}$ \\
\hline MONO [g/l] & $\begin{array}{c}2.58 \\
\pm 0.73\end{array}$ & $\begin{array}{c}2.37^{\mathrm{A}} \\
\pm 0.27\end{array}$ & $\begin{array}{c}3.00^{\mathrm{A}} \\
\pm 1.26\end{array}$ & $\begin{array}{c}1.88 \\
\pm 0.29\end{array}$ & $\begin{array}{c}2.86^{\mathrm{a}} \\
\pm 1.03\end{array}$ & $\begin{array}{l}1.38^{\mathrm{BB}} \\
\pm 0.79\end{array}$ & $\begin{array}{l}1.41^{\mathrm{BB}} \\
\pm 0.25\end{array}$ & $\begin{array}{c}2.04 \\
\pm 0.58\end{array}$ & $\begin{array}{c}2.25 \\
\pm 0.68\end{array}$ & $\begin{array}{l}2.12^{\mathrm{a}} \\
\pm 0.11\end{array}$ \\
\hline EOS $[g / l]$ & $\begin{array}{c}0.31 \\
\pm 0.06\end{array}$ & $\begin{array}{c}0.27 \\
\pm 0.03\end{array}$ & $\begin{array}{l}0.44^{\mathrm{A}} \\
\pm 0.15\end{array}$ & $\begin{array}{c}0.37 \\
\pm 0.11\end{array}$ & $\begin{array}{c}0.31 \\
\pm 0.11\end{array}$ & $\begin{array}{c}0.35 \\
\pm 0.19\end{array}$ & $\begin{array}{c}0.31 \\
\pm 0.09\end{array}$ & $\begin{array}{c}0.24 \\
\pm 0.02\end{array}$ & $\begin{array}{l}0.25^{\mathrm{B}} \\
\pm 0.07\end{array}$ & $\begin{array}{c}0.22 \\
\pm 0.09\end{array}$ \\
\hline BASO [g/l] & $\begin{array}{c}0.09 \\
\pm 0.05\end{array}$ & $\begin{array}{c}0.06 \\
\pm 0.03\end{array}$ & $\begin{array}{c}0.10 \\
\pm 0.04\end{array}$ & $\begin{array}{l}0.01^{b} \\
\pm 0.01\end{array}$ & $\begin{array}{l}0.11^{\mathrm{a}} \\
\pm 0.03\end{array}$ & $\begin{array}{c}0.07 \\
\pm 0.02\end{array}$ & $\begin{array}{l}0.11^{\mathrm{a}} \\
\pm 0.03\end{array}$ & $\begin{array}{c}0.09^{\mathrm{a}} \\
\pm 0.07\end{array}$ & $\begin{array}{l}0.04^{b} \\
\pm 0.05\end{array}$ & $\begin{array}{c}0.03 \\
\pm 0.005\end{array}$ \\
\hline $\mathrm{RBC}[\mathrm{T} / \mathrm{I}]$ & $\begin{array}{r}5.65^{\mathrm{B}} \\
\pm 0.29\end{array}$ & $\begin{array}{c}5.93 \\
\pm 0.37\end{array}$ & $\begin{array}{c}5.88 \\
\pm 0.31\end{array}$ & $\begin{array}{c}6.29 \\
\pm 0.22\end{array}$ & $\begin{array}{c}6.16 \\
\pm 0.53\end{array}$ & $\begin{array}{c}6.23 \\
\pm 0.35\end{array}$ & $\begin{array}{c}6.29 \\
\pm 0.63\end{array}$ & $\begin{array}{c}5.86 \\
\pm 0.32\end{array}$ & $\begin{array}{l}6.52^{\mathrm{A}} \\
\pm 0.47\end{array}$ & $\begin{array}{c}6.17 \\
\pm 0.38\end{array}$ \\
\hline HGB [g/l] & $\begin{array}{l}102.48 \\
\pm 5.64\end{array}$ & $\begin{array}{c}105.33^{\mathrm{aB}} \\
\pm 4.47\end{array}$ & $\begin{array}{l}107.00 \\
\pm 6.46\end{array}$ & $\begin{array}{c}113.50^{A} \\
\pm 2.88\end{array}$ & $\begin{array}{l}106.00 \\
\pm 5.55\end{array}$ & $\begin{array}{c}104.25^{\mathrm{B}} \\
\pm 4.74\end{array}$ & $\begin{array}{l}106.25 \\
\pm 3.89\end{array}$ & $\begin{array}{c}100.23^{\mathrm{bB}} \\
\pm 2.06\end{array}$ & $\begin{array}{l}109.75 \\
\pm 7.03\end{array}$ & $\begin{array}{c}104.75^{\mathrm{B}} \\
\pm 0.89\end{array}$ \\
\hline HCT [I/I] & $\begin{array}{l}0.33^{\mathrm{Bb}} \\
\pm 0.02\end{array}$ & $\begin{array}{l}0.35^{\mathrm{B}} \\
\pm 0.03\end{array}$ & $\begin{array}{l}0.36^{\mathrm{a}} \\
\pm 0.02\end{array}$ & $\begin{array}{l}0.39^{A} \\
\pm 0.02\end{array}$ & $\begin{array}{c}0.35 \\
\pm 0.03\end{array}$ & $\begin{array}{r}0.36^{\mathrm{B}} \\
\pm 0.01\end{array}$ & $\begin{array}{c}0.35 \\
\pm 0.02\end{array}$ & $\begin{array}{l}0.34^{\mathrm{B}} \\
\pm 0.01\end{array}$ & $\begin{array}{c}0.37^{\mathrm{A}} \\
\pm 0.02\end{array}$ & $\begin{array}{l}0.36^{\mathrm{B}} \\
\pm 0.01\end{array}$ \\
\hline MCV [pg] & $\begin{array}{r}57.70 \\
\pm 3.60\end{array}$ & $\begin{array}{l}59.75 \\
\pm 2.30\end{array}$ & $\begin{array}{l}61.10 \\
\pm 2.04\end{array}$ & $\begin{array}{l}62.45^{\mathrm{aA}} \\
\pm 0.70\end{array}$ & $\begin{array}{l}56.68 \\
\pm 2.44\end{array}$ & $\begin{array}{l}57.85^{\mathrm{B}} \\
\pm 2.09\end{array}$ & $\begin{array}{l}56.48 \\
\pm 4.67\end{array}$ & $\begin{array}{l}58.95^{\mathrm{b}} \\
\pm 2.44\end{array}$ & $\begin{array}{r}57.15 \\
\pm 2.45\end{array}$ & $\begin{array}{l}58.11^{\mathrm{B}} \\
\pm 2.74\end{array}$ \\
\hline MCH [g/l] & $\begin{array}{l}18.18^{a} \\
\pm 0.82\end{array}$ & $\begin{array}{l}17.80^{c} \\
\pm 0.61\end{array}$ & $\begin{array}{l}18.20^{\mathrm{a}} \\
\pm 0.42\end{array}$ & $\begin{array}{l}18.05^{\mathrm{aA}} \\
\pm 0.35\end{array}$ & $\begin{array}{r}17.25 \\
\pm 0.60\end{array}$ & $\begin{array}{c}16.75^{\mathrm{dB}} \\
\pm 0.19\end{array}$ & $\begin{array}{r}17.10 \\
\pm 1.19\end{array}$ & $\begin{array}{l}17.13 \\
\pm 0,49\end{array}$ & $\begin{array}{l}16.88^{\mathrm{b}} \\
\pm 0.63\end{array}$ & $\begin{array}{l}17.00^{b} \\
\pm 1.16\end{array}$ \\
\hline MCHC [g/l] & $\begin{array}{c}314.25^{\mathrm{aA}} \\
\pm 3.37\end{array}$ & $\begin{array}{c}298.00^{\mathrm{a}} \\
\pm 4.11\end{array}$ & $\begin{array}{c}298.00^{B} \\
\pm 5.29\end{array}$ & $\begin{array}{c}288.75^{b} \\
\pm 3.45\end{array}$ & $\begin{array}{l}304.50 \\
\pm 6.99\end{array}$ & $\begin{array}{c}290.00^{\mathrm{b}} \\
\pm 9.86\end{array}$ & $\begin{array}{l}302.25^{b} \\
\pm 11.69\end{array}$ & $\begin{array}{l}290.50 \\
\pm 1.38\end{array}$ & $\begin{array}{c}295.75^{\mathrm{B}} \\
\pm 5.90\end{array}$ & $\begin{array}{l}292.25 \\
\pm 4.33\end{array}$ \\
\hline PLT [g/l] & $\begin{array}{c}366.15 \\
\pm 202.08\end{array}$ & $\begin{array}{c}278.75 \\
\pm 126.91\end{array}$ & $\begin{array}{c}392.68 \\
\pm 203.62\end{array}$ & $\begin{array}{c}189.98 \\
\pm 165.75\end{array}$ & $\begin{array}{c}398.75 \\
\pm 114.40\end{array}$ & $\begin{array}{c}222.85 \\
\pm 142.83\end{array}$ & $\begin{array}{c}230.93 \\
\pm 109.97\end{array}$ & $\begin{array}{r}231.75 \\
\pm 52.90\end{array}$ & $\begin{array}{c}187.85 \\
\pm 118.95\end{array}$ & $\begin{array}{c}275.65 \\
\pm 154.17\end{array}$ \\
\hline
\end{tabular}

Explanation: as in Tab. 4. 
average level of NEU was statistically more different in the $8^{\text {th }}$ week of life. The lowest concentration of LYM was observed in both control terms in Group C, which was significantly different in this respect from Groups $\mathrm{B}+, \mathrm{B}-$ and $\mathrm{A}-(\mathrm{P} \leq 0.01$ and $\mathrm{P} \leq 0.05)$. The level of MONO in the first term was lowest in Group $\mathrm{A}+$, and it was significantly different from that in Groups $\mathrm{B}+$ and $\mathrm{B}-(\mathrm{P} \leq 0.01$ and $\mathrm{P} \leq 0.05)$. In the second term, the lowest level of MONO occurred in group $\mathrm{B}-$, and it was significantly different from MONO levels in Groups $\mathrm{C}$ and $\mathrm{A}-(\mathrm{P} \leq 0.01$ and $\mathrm{P} \leq 0.05)$. With regard to red cell indices in the second control term, statistically significant differences were noted in HGB and HTC, which were the highest in Group B+. The highest value of MCV was found in Group B+, and it was significantly different from MCV values in Groups B-, $\mathrm{A}-$ and $\mathrm{A}+(\mathrm{P} \leq 0.01$ and $\mathrm{P} \leq 0.05)$. High statistical differences between groups in $\mathrm{MCH}$ and $\mathrm{MCHC}$ were observed in both control terms.

The average concentration of TP in the serum of sows 3 weeks before delivery was $70.36 \mathrm{~g} / \mathrm{L}$, and the difference of $9 \mathrm{~g} / \mathrm{l}$ between Groups $\mathrm{C}$ and $\mathrm{A}$ was im-

Tab. 8. Total protein and its fractions in sow serum 3 weeks before delivery $(\bar{x} \pm$ SD)

\begin{tabular}{|l|c|c|c|}
\hline \multicolumn{1}{|c|}{ Parameter } & Group C & Group B & Group A \\
\hline Total protein [g/l] & $74.61^{\mathrm{a}}$ & 70.90 & $65.58^{\mathrm{b}}$ \\
& \pm 6.08 & \pm 6.06 & \pm 6.62 \\
Albumin [g/l] & 23.42 & 25.41 & 26.00 \\
& \pm 1.21 & \pm 3.39 & \pm 1.91 \\
a-globulin [g/l] & $14.07^{\text {Aa }}$ & $11.79^{\mathrm{b}}$ & $10.55^{\mathrm{B}}$ \\
& \pm 1.93 & \pm 1.87 & \pm 1.42 \\
$\beta$ & $14.96^{\mathrm{a}}$ & $11.98^{\mathrm{b}}$ & 12.63 \\
& \pm 2.85 & \pm 1.04 & \pm 2.41 \\
$\gamma$-globulin [g/l] & $22.21^{\mathrm{a}}$ & 21.11 & $17.05^{\mathrm{b}}$ \\
& \pm 3.54 & \pm 3.11 & \pm 3.89 \\
Albumin/globulin & $0.46^{\mathrm{Bb}}$ & $0.56^{\mathrm{ad}}$ & $0.67^{\mathrm{Ac}}$ \\
& \pm 0.04 & \pm 0.08 & \pm 0.08 \\
\hline
\end{tabular}

Explanation: as in Tab. 4. portant (Tab. 8). Statistically meaningful differences were noted in globulin fractions between Group C (in which they were the highest) and the other groups. The lowest value of $\mathrm{A} / \mathrm{G}$ was noted in the control group, and this result differed statistically from results in Groups $\mathrm{A}(\mathrm{P} \leq 0.01)$ and $\mathrm{B}(\mathrm{P} \leq 0.05)$; a significant difference was also confirmed between groups $\mathrm{B}$ and $\mathrm{A}(\mathrm{P} \leq 0.05)$.

The average value of TP and its fractions in piglets' and weaners' serum is shown in Tables 9 and 10. In the first control term, the highest concentration of TP was found in the control group, and the difference of $7 \mathrm{~g} / 1$ between the control group and Group A+ was statistically important. In the subsequent control terms, the concentration of TP in Group C was the lowest, and in the $3^{\text {rd }}$ week of life the differences between Group $\mathrm{C}$ and Groups B+ and $\mathrm{A}+$ were statistically confirmed. A similar tendency was observed for albumin concentration.

An analysis of body weight changes during the experimental period allowed us to conclude that growth in all pigs was comparatively high $(11,20,30)$, and except for the suckling period, the growth rate in the experimental groups was distinctly higher than it was in the control group. Usually the positive effect of probiotics on growth is reported in suckling and weaned piglets $(15,23)$ and rarely in fatteners $(3,26)$. Many authors $(16,23)$ report that probiotics, as well as garlic preparations, could be used as growth stimulants, but in older healthy pigs no noteworthy effects were usually observed. In the present trial, the use of both feed additives made it possible to obtain a satisfactory final body weight of fatteners. It is important for pig producers because this can shorten the occupation of the facilities, thus leading to better productivity and potentially higher income. Tatara et al. studied the effect of perinatal administration of aged garlic extract (AGE) and allicin (ALL) to pregnant sows on the growth and development of piglets. Sows were given AGE or allicin daily from the $91^{\text {st }}$ day of pregnancy

Tab. 9. Total protein and its fractions in piglet serum $(\overline{\mathbf{x}} \pm \mathrm{SD})$

\begin{tabular}{|c|c|c|c|c|c|c|c|c|c|}
\hline \multirow[t]{2}{*}{ Parameter } & \multicolumn{3}{|c|}{ Group C } & \multicolumn{3}{|c|}{$\begin{array}{c}\text { Group B+ } \\
\text { Week of life }\end{array}$} & \multicolumn{3}{|c|}{ Group A+ } \\
\hline & $1^{\text {st }}$ & $3^{\text {rd }}$ & $4^{\text {th }}$ & $1^{s t}$ & $3^{\text {rd }}$ & $4^{\text {th }}$ & $1^{\text {st }}$ & $3^{\text {rd }}$ & $4^{\text {th }}$ \\
\hline Total protein [g/l] & $\begin{array}{l}55.09 \mathrm{a} \\
\pm 6.15\end{array}$ & $\begin{array}{l}41.67^{\mathrm{B}} \\
\pm 4.82\end{array}$ & $\begin{array}{l}47.45 \\
\pm 5.72\end{array}$ & $\begin{array}{r}49.08 \\
\pm 5.86\end{array}$ & $\begin{array}{l}55.65^{\mathrm{A}} \\
\pm 5.79\end{array}$ & $\begin{array}{r}47.88 \\
\pm 3.05\end{array}$ & $\begin{array}{l}48.00^{b} \\
\pm 2.85\end{array}$ & $\begin{array}{l}53.87^{\mathrm{A}} \\
\pm 7.49\end{array}$ & $\begin{array}{r}49.57 \\
\pm 3.00\end{array}$ \\
\hline Albumin [g/l] & $\begin{array}{r}17.20 \\
\pm 1.89\end{array}$ & $\begin{array}{l}19.47^{\mathrm{b}} \\
\pm 1.95\end{array}$ & $\begin{array}{l}23.25 \\
\pm 3.24\end{array}$ & $\begin{array}{r}14.50 \\
\pm 2.39\end{array}$ & $\begin{array}{l}22.78^{a} \\
\pm 2.58\end{array}$ & $\begin{array}{r}24.87 \\
\pm 1.65\end{array}$ & $\begin{array}{r}15.49 \\
\pm 2.54\end{array}$ & $\begin{array}{r}22.40 \\
\pm 2.55\end{array}$ & $\begin{array}{r}24.18 \\
\pm 1.87\end{array}$ \\
\hline$\alpha$-globulin [g/l] & $\begin{array}{r}10.58 \\
\pm 0.82\end{array}$ & $\begin{array}{c}9.65^{\mathrm{B}} \\
\pm 1.39\end{array}$ & $\begin{array}{l}11.46^{\mathrm{Aa}} \\
\pm 1.12\end{array}$ & $\begin{array}{c}9.99 \\
\pm 1.73\end{array}$ & $\begin{array}{l}14.79^{\mathrm{A}} \\
\pm 4.81\end{array}$ & $\begin{array}{c}7.24^{\mathrm{B}} \\
\pm 1.16\end{array}$ & $\begin{array}{c}9.83 \\
\pm 1.65\end{array}$ & $\begin{array}{c}7.94^{\mathrm{B}} \\
\pm 1.12\end{array}$ & $\begin{array}{c}9.15^{b} \\
\pm 2.13\end{array}$ \\
\hline$\beta$-globulin [g/l] & $\begin{array}{r}11.17 \\
\pm 1.39\end{array}$ & $\begin{array}{c}8.00^{\mathrm{B}} \\
\pm 1.54\end{array}$ & $\begin{array}{c}7.21 \\
\pm 1.47\end{array}$ & $\begin{array}{l}10.01 \\
\pm 3.09\end{array}$ & $\begin{array}{c}8.89^{\mathrm{B}} \\
\pm 1.53\end{array}$ & $\begin{array}{c}9.85 \\
\pm 1.14\end{array}$ & $\begin{array}{r}10.05 \\
\pm 1.48\end{array}$ & $\begin{array}{l}12.30^{A} \\
\pm 2.38\end{array}$ & $\begin{array}{r}10.29 \\
\pm 1.68\end{array}$ \\
\hline$\gamma$-globulin [g/l] & $\begin{array}{r}16.14 \\
\pm 4.05\end{array}$ & $\begin{array}{c}4.51^{\mathrm{b}} \\
\pm 2.11\end{array}$ & $\begin{array}{c}5.51 \\
\pm 1.39\end{array}$ & $\begin{array}{r}14.59 \\
\pm 1.18\end{array}$ & $\begin{array}{c}9.42 \\
\pm 1.46\end{array}$ & $\begin{array}{c}5.91 \\
\pm 0.89\end{array}$ & $\begin{array}{r}12.65 \\
\pm 3.85\end{array}$ & $\begin{array}{l}10.94^{\mathrm{a}} \\
\pm 7.48\end{array}$ & $\begin{array}{c}5.95 \\
\pm 1.73\end{array}$ \\
\hline Albumin/globulin & $\begin{array}{c}0.46 \\
\pm 0.07\end{array}$ & $\begin{array}{c}0.80 \\
\pm 0.31\end{array}$ & $\begin{array}{c}0.96 \\
\pm 0.07\end{array}$ & $\begin{array}{c}0.42^{\mathrm{b}} \\
\pm 0.05\end{array}$ & $\begin{array}{c}0.70 \\
\pm 0.18\end{array}$ & $\begin{array}{c}1.08 \\
\pm 0.04\end{array}$ & $\begin{array}{c}0.50^{\mathrm{a}} \\
\pm 0.08\end{array}$ & $\begin{array}{c}0.75 \\
\pm 0.27\end{array}$ & $\begin{array}{c}0.97 \\
\pm 0.19\end{array}$ \\
\hline
\end{tabular}

Explanation: as in Tab. 4. 
Tab. 10. Total protein and its fractions in weaner serum $(\overline{\mathbf{x}}$ \pm SD)

\begin{tabular}{|c|c|c|c|c|c|}
\hline Parameter & Group C & Group B+ & Group B- & Group A+ & Group A- \\
\hline & \multicolumn{5}{|c|}{$8^{\text {th }}$ week of life } \\
\hline Total protein [g/l] & $\begin{array}{l}40.62 \\
\pm 2.77\end{array}$ & $\begin{array}{r}41.43 \\
\pm 3.64\end{array}$ & $\begin{array}{r}41.83 \\
\pm 2.02\end{array}$ & $\begin{array}{r}41.23 \\
\pm 1.78\end{array}$ & $\begin{array}{r}41.43 \\
\pm 0.62\end{array}$ \\
\hline Albumin $[g / l]$ & $\begin{array}{l}16.45^{\mathrm{B}} \\
\pm 1.81\end{array}$ & $\begin{array}{r}18.63 \\
\pm 3.87\end{array}$ & $\begin{array}{r}18.90 \\
\pm 1.54\end{array}$ & $\begin{array}{r}18.60 \\
\pm 0.85\end{array}$ & $\begin{array}{l}20.72^{\mathrm{A}} \\
\pm 0.78\end{array}$ \\
\hline$\alpha$-globulin [g/l] & $\begin{array}{c}9.75^{\mathrm{A}} \\
\pm 1.28\end{array}$ & $\begin{array}{r}9.76^{\mathrm{A}} \\
\pm 1.09\end{array}$ & $\begin{array}{c}8.80 \\
\pm 0.82\end{array}$ & $\begin{array}{c}8.60 \\
\pm 0.74\end{array}$ & $\begin{array}{r}7.91^{\mathrm{B}} \\
\pm 0.39\end{array}$ \\
\hline$\beta$-globulin [g/l] & $\begin{array}{c}8.44 \\
\pm 1.53\end{array}$ & $\begin{array}{c}8.26 \\
\pm 0.62\end{array}$ & $\begin{array}{c}8.80 \\
\pm 0.82\end{array}$ & $\begin{array}{c}9.15 \\
\pm 0.93\end{array}$ & $\begin{array}{c}8.06 \\
\pm 0.49\end{array}$ \\
\hline$\gamma$-globulin [g/l] & $\begin{array}{c}6.06 \\
\pm 2.20\end{array}$ & $\begin{array}{c}5.72 \\
\pm 3.66\end{array}$ & $\begin{array}{c}4.74 \\
\pm 0.92\end{array}$ & $\begin{array}{c}4.89 \\
\pm 0.44\end{array}$ & $\begin{array}{c}4.73 \\
\pm 0.86\end{array}$ \\
\hline \multirow[t]{2}{*}{ Albumin/globulin } & $\begin{array}{c}0.69^{b} \\
\pm 0.22\end{array}$ & $\begin{array}{c}0.83 \\
\pm 0.30\end{array}$ & $\begin{array}{c}0.84 \\
\pm 0.30\end{array}$ & $\begin{array}{c}0.83 \\
\pm 0.06\end{array}$ & $\begin{array}{r}1.00^{\mathrm{a}} \\
\pm 0.09\end{array}$ \\
\hline & \multicolumn{5}{|c|}{$11^{\text {th }}$ week of life } \\
\hline Total protein [g/l] & $\begin{array}{l}45.14 \\
\pm 2.39\end{array}$ & $\begin{array}{l}48.61 \\
\pm 3.32\end{array}$ & $\begin{array}{r}44.95 \\
\pm 5.74\end{array}$ & $\begin{array}{r}43.40 \\
\pm 5.33\end{array}$ & $\begin{array}{l}47.17 \\
\pm 2.79\end{array}$ \\
\hline Albumin $[g / l]$ & $\begin{array}{r}17.40 \\
\pm 0.85\end{array}$ & $\begin{array}{l}21.87^{\mathrm{a}} \\
\pm 3.97\end{array}$ & $\begin{array}{r}19.28 \\
\pm 3.32\end{array}$ & $\begin{array}{l}17.02^{b} \\
\pm 4.42\end{array}$ & $\begin{array}{r}19.93 \\
\pm 1.28\end{array}$ \\
\hline$\alpha$-globulin [g/l] & $\begin{array}{c}6.62 \\
\pm 0.56\end{array}$ & $\begin{array}{r}12.98 \\
\pm 1.44\end{array}$ & $\begin{array}{r}14.18 \\
\pm 3.34\end{array}$ & $\begin{array}{r}13.80 \\
\pm 4.56\end{array}$ & $\begin{array}{r}13.25 \\
\pm 2.25\end{array}$ \\
\hline$\beta$-globulin [g/l] & $\begin{array}{c}6.62 \\
\pm 0.56\end{array}$ & $\begin{array}{c}7.60 \\
\pm 1.57\end{array}$ & $\begin{array}{c}6.62 \\
\pm 0.67\end{array}$ & $\begin{array}{c}6.31 \\
\pm 3.18\end{array}$ & $\begin{array}{c}7.12 \\
\pm 1.51\end{array}$ \\
\hline$\gamma$-globulin [g/l] & $\begin{array}{c}8.55 \\
\pm 2.22\end{array}$ & $\begin{array}{c}6.17 \\
\pm 3.82\end{array}$ & $\begin{array}{c}4.90 \\
\pm 1.09\end{array}$ & $\begin{array}{c}6.24 \\
\pm 1.77\end{array}$ & $\begin{array}{c}6.87 \\
\pm 3.65\end{array}$ \\
\hline Albumin/globulin & $\begin{array}{c}0.63 \\
\pm 0.13\end{array}$ & $\begin{array}{c}0.83 \\
\pm 0.22\end{array}$ & $\begin{array}{c}0.76 \\
\pm 0.09\end{array}$ & $\begin{array}{c}0.65 \\
\pm 0.30\end{array}$ & $\begin{array}{c}0.73 \\
\pm 0.09\end{array}$ \\
\hline
\end{tabular}

Explanation: as in Tab. 4.

up to weaning on the $28^{\text {th }}$ day of piglets' life. Those author's results confirmed that the administration of the garlic preparations to the mother improved nutrition at early stages of postnatal development and had positive effects on body weight determined at birth and during 56 days of postnatal development of piglets. Similarly, our results showed a relationship between the garlic extract and improved weight gain compared to that in the control group. On the $3^{\text {rd }}$ and $56^{\text {th }}$ days of piglets' life, Tatara's experimental groups were heavier than the control group by respectively $0.5 \mathrm{~kg}$ and $4.6 \mathrm{~kg}$ on average (32). In our study, piglets in group A+ (receiving garlic) were heavier than the control group on the $2^{\text {nd }}$ and $56^{\text {th }}$ days of life by $0.03 \mathrm{~kg}$ and $4 \mathrm{~kg}$, respectively.

With regard to bacteria supplementation, Delia et al. (9) conducted a trial under extensive pig farming conditions. After weaning, piglets were fed for 8 weeks with a basic feed enriched with 1000, 1500 and 2000 $\mathrm{mg} / \mathrm{kg}$ of probiotic treatment. This probiotic preparation contained Lactobacillus plantarium ATCC 14917 $1 \times 10^{11} \mathrm{cfu} / \mathrm{kg}$, Lactobacillus fermentum DSM 20016 $1 \times 10^{11} \mathrm{cfu} / \mathrm{kg}$ and Enterociccus faecium ATCC 19434 $1 \times 10^{11} \mathrm{cfu} / \mathrm{kg}$. In the above study, a significant difference in daily weight gain (DWG) was documented four weeks after probiotic supplementation. After 56 days, pigs supplemented with $1000 \mathrm{mg} / \mathrm{kg}$ of probiotic preparation were heavier by $3.11 \mathrm{~kg}$ than the control group. This was consistent with the results of our trial, in which the most noticeable differences in piglets' growth appeared after weaning.

The average white blood cell counts in sows from all experimental groups were within the range of reference values given by Elbers et al. (12). However, the lowest count of WBC among all sub-populations of sows 3 weeks before delivery was found in Group A, which received the garlic extract. It may indicate their better health status. This result correlates with that from a study by Kaiser et al. (19), who found that healthy sows expressed lower values of WBC and NEU than sows with diagnosed dysgalactia syndrome. And this observation coincides with the interpretation of the concentration of globulin fraction presented below.

The average concentration of TP in all sows was satisfactory according to recommendations from Elbers et al. (12) and Verheyen et al. (37). Serum albumin could be used as a predictor of the nutritional status in animals, and its slightly higher concentration in experimental groups may suggest a better feed digestibility - especially in connection with a lower concentration of all globulin fractions, which can attest to the improved health status of sows in the trial. This observation results from an evaluation of the alb/glob value. However, the average values are generally low (37). The lowest value was noted in the control sows, which can be explained by the greater risk of bacterial infection in this group.

Our findings concerning the influence of probiotic supplementation on pigs' haematology show that Group B + had the lowest levels of NEU and BASO in the $4^{\text {th }}$ week of life. This result is similar to that presented by Dlamini et al. (10), who described the beneficial effects of a probiotic bacteria mixture given to pigs from the $4^{\text {th }}$ to the $8^{\text {th }}$ week of life. The abovementioned authors suggest that a high level of NEU and BASO may be a sign of an ongoing infection, which in our study correlates with results from the control group. In our study, the levels of WBC and LYM in Group $\mathrm{A}+$ at weaning were lower than in the control group, but higher than in Group $\mathrm{B}+$. In contrast, in a study by Yan and Kim (39) concerning the use of garlic, the concentration of WBC and LYM increased after its administration. These authors suppose that this may have been due to increased phagocytic function in macrophage and lymphocyte proliferation or to overstimulation. Dlamini et al. (10) showed that piglets supplemented with probiotics had a higher concentration of IgG. They believe that probiotic bacteria can act on the immune system as an adjuvant and enhance IgG production. Our results could confirm this, but we also suggest another explanation. The higher concentration of globulins in piglets supplemented with probiotics could have resulted from the immunoprotective proper- 
ties of this additive. However, our hypothesis is that, because of the presence of the additional probiotic bacteria which take part in the host's defence, the immune system of piglets may not be stimulated or may be slightly stimulated to develop an active immune response.

The concentration of TP and its fraction in the first week of a piglet's life can indicate strong protection imparted by maternal immunity. Our results show convergence with those of Szymeczko et al. (31), particularly with regard to the control group. Around the $3^{\text {rd }}$ week of life, when a peak of milk yield can usually be expected (18) along with a decrease in piglets' passive immunity, a higher concentration of TP was observed in Groups A+ and B+, and it differed significantly from that in the control group. Moreover, these animals had a higher concentration of ALB, which can be explained by a better conversion of feed and milk. On the other hand, piglets from the control group were not additionally protected by feed additives, and they had the lowest $\gamma$-globulin concentration and the highest decrease in this fraction. Tatara et al. (33) investigated the effects of postnatal administration of aged garlic extract (AGE) and allicin on the performance and development of the piglets' gastrointestinal tract (GI). They concluded that AGE and allicin given postnatally to piglets helped in the development of GI and supported an immunostimulating effect on non-specific defence mechanisms. In our study, during the weaning time, protein electrophoresis results were more equal in all population. However, the higher level of $\alpha$-globulin fraction in Group $\mathrm{C}$ may suggest development of new inflammations or infections because this fraction contains, among others, acute-phase proteins (APPs) that appear at the beginning of such disturbances $(8,35)$.

Determination of blood parameters, such as proteinogram and morphology, may be useful in pigs in detecting inflammation and unfavourable health conditions or in confirming the absence of health disorders as a result of a good animal management and protection programme $(8,26)$. Decreased values of serum albumin, leucocytosis and increased gamma-globulin levels indicate an ongoing inflammatory process (13). When comparing these results with blood parameters of fatteners in our study, the least favourable results were obtained in the control group and in group A+ (supplemented with the garlic extract). It seems that the effect of probiotics on blood indicators is best when they are administered until the $8^{\text {th }}$ week of piglets' life, especially in terms of albumin and WBC levels.

The results indicate that supplementation with a garlic extract and probiotic bacteria is most beneficial when it lasts until the $8^{\text {th }}$ week of a pig's life, for both economic and health reasons.

To the best of our knowledge, this is the first study in which the efficacy of a garlic extract and probiotics was evaluated simultaneously, in the same field trial and under the same environmental farm conditions. A distinct relationship between pigs' body weight and blood parameters, influenced by the feed additives, was found under field conditions. The results suggest that the use of the garlic extract and the probiotic bacteria Enterococcus faecium, Lactobacillus rhamnosus and Lactobacillus fermentum may help improve pigs' performance at various stages of the production cycle. Supplementation of these additives to piglets until the $56^{\text {th }}$ day of their life improved their average body weight. Continued supplementation of garlic and probiotics (up to 147 days) does not seem to have any notable effect. Animals from groups supplemented for a longer period $(\mathrm{A}+, \mathrm{B}+)$ were not heavier than those that received both additives only until the $56^{\text {th }}$ day of life. At the end of the fattening period, the control pigs were lighter by $15 \mathrm{~kg}$ than the others.

Since the growth rate of animals differs strongly between the growing and fattening periods, the ultimate determination of the optimal time for supplementing these additives may require further studies.

\section{References}

1. Ahasan A. S. M. L., Agazzi A., Invernizzi G., Bontempo V., Savoini G.: The beneficial role of probiotics in monogastric animal nutrition and health. JDVAR 2015, 2, 116-132, doi: 10.15406/jdvar.2015.02.00041.

2. Ahmed S. T., Hwang J. A., Hoon J., Mun H. S., Yang C. J.: Comparison of single and blend acidifiers as alternative to antibiotics on growth performance, fecal microflora, and humoral immunity in weaned piglets. Asian-Australasian J. Anim. Sci. 2014, 27, 93-100, doi: 10.5713/ajas.2013.13411.

3. Balasubramanian B., Li T., Kim I. H.: Effects of supplementing growingfinishing pig diets with Bacillus spp. probiotic on growth performance and meat-carcass grade quality traits. R. Bras. Zootec. 2016, 45, 93-100.

4. Barba-Vidal E., Martín-Orúe S. M., Castillejos L.: Review: Are we using probiotics correctly in post-weaning piglets? Animal 2018, 12, 2489-2498, doi: $10.1017 / \mathrm{S} 1751731118000873$.

5. Bayan L., Koulivand P. H, Gorji A.: Garlic: a review of potential therapeutic effects, Avicenna J. Phytomed. 2014, 4, 1-14.

6.Brzozowski B., Bednarski, W., Golek P.: The adhesive capability of two Lactobacillus strains and physicochemical properties of their synthesized biosurfactants. Food Technol. Biotechnol. 2011, 49, 177-186.

7. Cai C. J., Cai P. P., Hou C. L., Zeng X. F., Qiao S. Y.: Administration of Lactobacillus fermentum I5007 to young piglets improved their health and growth. J. Anim. Feed Sci. 2014, 23, 222-227.

8. Cray C., Zaias J., Altman N. H.: Acute phase response in animals: a review. Comparative Med. 2009, 59, 517-526.

9. Delia E., Tafaj M., Manner K.: Efficiency of probiotics in farm animals. Intech. 2012, 11, 256-267.

10. Dlamini Z. C., Langa R. L. S., Aiyegoro O. A., Okoh A. I.: Effects of probiotics on growth performance, blood parameters, and antibody stimulation in piglets. S. Afr. J. Anim. Sci. 2017, 47, 766-775.

11. Dritz S. S., Owen K. Q., Nelssen J. L., Goodband R. D., Tokach M. D.: Influence of weaning age and nursery diet complexity on growth performance and carcass characteristic and composition of high-health status pigs from weaning to 109 kilograms. J. Anim. Sci. 1996, 74, 2975-2984.

12. Elbers A. R. W., Geudeke M. J., Rossen H. van, Kroon M. C., Counotte C. H. M. Haematology and biochemistry reference values for sows kept under modern management conditions. Vet. Quart. 1994, 16, 127-130.

13. Elbers A. R. W., Visser I. J. R., Odink J., Smeets J. F. M.: Changes in haematological and clinicochemical profiles in blood of apparently healthy slaughter pigs, collected at the farm and at slaughter, in relation to the severity of pathological-anatomical lesions. Vet. Quart. 1991, 13, 1-9, doi: 10.1080/01652176.1991.9694278.

14. Gheisar M. M., Kim I. H.: Phytobiotics in poultry and swine nutrition a review. Ital. J. Anim. Sci. 2017, 17, 92-99.

15. Giang H. H., Viet T. Q., Ogle B., Lindberg J. E.: Effects of different probiotic complexes of lactic acid bacteria on growth performance and gut environment of weaned piglets. Livest. Sci. 2010, 133, 182-184. 
16. Grela E. R., Krusiński R., Matras J.: Efficacy of diets with antibiotic and herb mixture additives in feeding of growing-finishing pigs. J. Anim. Feed Sci. 1998, 7, 171-175.

17. Hanczakowska E., Swiatkiewicz M.: Effect of herbal extracts on piglet performance and small intestinal epithelial villi. Czech J. Anim. Sci. 2012, 57, 420-429.

18. Hansen A. V., Strathe A. B., Kebreab E., France J., Theil P. K.: Predicting milk yield and composition in lactating sow: A Bayesian approach. J. Anim. Sci. 2016, 90, 2285-2298.

19. Kaiser M., Jacobson M., Andersen P. H., Bakbo P., Cerón J. J., Dahl J. Jacobsen $S$. : Inflammatory markers before and after farrowing in healthy sows and in sows affected with postpartum dysgalactia syndrome. BMC Vet. Res. 2018, 14, 83, doi: 10.1186/s12917-018-1382-7.

20. Kim S. W., Hurley W. L., Hant I. K., Easter R. A.: Growth of nursing pigs related to the characteristics of nursed mammary glands. J. Anim. Sci. 2000, 78, 1313-1318.

21. Kritas S. K., Morrison R. B.: Evaluation of probiotics as substitute for antibiotics in a large pig nursery. Vet. Rec. 2005, 156, 447-448.

22. Lillehoj H., Liu Y., Calsamiglia S., Fernandez-Miyakawa M. E., Chi F., Cravens R. L., Oh S., Cyril G., Gay C. G.: Phytochemicals as antibiotic alternatives to promote growth and enhance host health. Vet. Res. 2018, 49, 76-93, doi: 10.1186/s13567-018-0562-6.

23. Link R., Kováč G., Pistl J.: A note on probiotics as an alternative for antibiotics in pigs. J. Anim. Feed Sci. 2005, 14, 513-519.

24. Omonijo F. A., Ni L., Gong J., Wang Q., Lahaye L., Yang C.: Essential oils as alternatives to antibiotics in swine production. Anim. Nutr. 2018, 4, 126-136.

25. Regulation EC No. 1831/2003 of the European Parliament and of the Council of 22 September 2003 on additives for use in animal nutrition.

26. Rzasa A., Stefaniak T., Jawor P., Gosiewski S., Toborowicz P.: The effect of HERB-mix ${ }^{R}$ in piglets' diarrhea prevention. Arch. Tierz. Dummerstorf 50. 2007, Special Issue, 136-143.

27. Skaradzińska A., Śliwka P., Kuźmińska-Bajor M., Skaradziński G., Rząsa A., Friese A., Roschanski N., Murugaiyan J., Roesler U. H.: The efficacy of isolated bacteriophages from pig farms against ESBL/AmpC-Producing Escherichia coli from pig and turkey farms. Front. Microbiol. 2017, doi:10.3389/fmicb.2017.00530.

28. Stefaniak T., Rzasa A., Jawor P., Zyzak A., Niemczuk W., Kuczaj M., Popławski M., Borkowski J.: Field application of egg yolk immunoglobulin as the feed additive in prophylaxis of diseases in weaned piglets. Med. Weter. 2014, 70, 553-557.

29. Sugiharto S., Riis Poulsen A.-S., Canibe N., Lauridsen C.: Effect of bovine colostrum feeding in comparison with milk replacer and natural feeding on the immune responses and colonisation of enterotoxigenic Escherichia coli in the intestinal tissue of piglets. Br. J. Nutr. 2015, 113, 923-934, doi: 10.1017 S0007114514003201.
30. Szuba-Trznadel A., Rząsa A., Lira R., Fuchs B.: The influence of $(1,3)-(1,6)-\beta-D-$ glucan on the production results of sows and their offspring. J. Anim. Feed Sci. 2014, 23, 228-235.

31. Szymeczko R., Kapelański W., Piotrowska A., Dybała J., Bogusławska-TrykM., Burlikowska K., Hertig I., Sassek M., Pruszyńska-Oszmałek E., Maćkowiak P. Changes in the Content of Major Proteins and Selected Hormones in the Blood Serum of Piglets During the Early Postnatal Period. Folia Biol. (Krakow) 2009, 1, 97-103, doi: 10.3409/fb57 1-2.97-1034

32. Tatara M. R., Śliwa E., Dudek K., Mosiewicz J., Studziński T.: Effect of aged garlic extract and allicin administration to sows during pregnancy and lactation on body weight gain and gastrointestinal tract development of piglets. Part I. Bull. Vet. Inst. Pulawy 2005, 49, 349-355.

33. Tatara M. R., Tomaszewska E., Dudek K., Gawron A., Piersiak T., Dobrowolski P., Mosiewicz J., Siwicki K. A., Studzinski T.: Aged garlic extract and allicin improve performance and gastrointestinal tract development of piglets reared in artificial sow. Annals of agricultural and environmental medicine. AAEM 2008, 15, 63-69

34. Thacker $P$. A.: Alternatives to antibiotics as growth promoters for use in swine production: a review. J. Anim. Sci. Biotechnol. 2013, 4, 35-46, doi: 10.1186/2049-1891-4-35.

35. Tothova C., Nay O., Kováč G.: Serum proteins and their diagnostic utility in veterinary medicine: a review. Vet. Med.-Czech. 2016, 61, 475-496.

36. Turner J. L., Dritz S. S., Minton J. E.: Alternatives to conventional antimicrobials in swine diets. PAS 2001, 17, 217-226.

37. Verheyen A. J., Maes D. G., Mateusen B., Deprez P., Janssens G. P., Lange L. de, Counotte $G$.: Serum biochemical reference values for gestating and lactating sows. Vet. J. 2007, 174, 92-98, doi: 10.1016/j.tvj1.2006.04.001.

38. Wang Y., Cho J. H., Chen Y. J., Yoo J. S., Huang Y., Kim H. J., Kim I. H.: The effect of probiotic BioPlus ${ }^{\circledR}$ on growth performance, dry matter and nitrogen digestibility and slurry noxious gas emission in growing pigs. Livest. Sci. 2009, 120, 35-42.

39. Yan L., Kim I. H.: Effects of dietary supplementation of fermented garlic powder on growth performance, apparent total tract digestibility, blood characteristics and faecal microbial concentration in weanling pigs. J. Anim. Physiol. Anim. Nutr. 2013, 97, 457-464.

40. Zeyner A., Boldt E.: Effects of probiotic Enterococcus faecium strain supplemented from birth to weaning on diarrhoea patterns and performance of piglets J. Anim. Physiol. Anim. Nutr. 2006, 90, 25-31.

Corresponding author: DVM, PhD, Prof. Univ. Katarzyna PloneczkaJaneczko, Department of Epizootiology and Clinic for Birds and Exotic Animals, Faculty of Veterinary Medicine, Wroclaw University of Environmental and Life Sciences, Wroclaw, Poland; e-mail: katarzyna.ploneczkajaneczko@upwr.edu.pl 